\title{
THE TIGHT RELATION BETWEEN X-RAY AND ULTRAVIOLET LUMINOSITY OF QUASARS
}

\author{
E. Lusso ${ }^{1}$ AND G. RISALITI ${ }^{2,1}$ \\ ${ }^{1}$ INAF-Osservatorio Astrofisico di Arcetri, I-50125 Florence, Italy; lusso@arcetri.astro.it
${ }^{2}$ Dipartimento di Fisica e Astronomia, Università di Firenze, via G. Sansone 1, 50019 Sesto Fiorentino (Firenze), Italy \\ Received 2015 November 2; accepted 2016 January 29; published 2016 March 8
}

\begin{abstract}
The observed relation between the soft X-ray and the optical-ultraviolet emission in active galactic nuclei (AGNs) is nonlinear and it is usually parametrized as a dependence between the logarithm of the monochromatic luminosity at $2500 \AA$ and at $2 \mathrm{keV}$. Previous investigations have found that the dispersion of this relation is rather high ( 0.35-0.4 in log units), which may be caused by measurement uncertainties, variability, and intrinsic dispersion due to differences in the AGN physical properties (e.g., different accretion modes). We show that, once optically selected quasars with homogeneous SED and X-ray detection are selected, and dust reddened and/or gas obscured objects are not included, the measured dispersion drops to significantly lower values (i.e., $\sim 0.21-0.24$ dex). We show that the residual dispersion is due to some extent to variability, and to remaining measurement uncertainties. Therefore, the real physical intrinsic dispersion should be $<0.21$ dex. Such a tight relation, valid over four decades in luminosity, must be the manifestation of an intrinsic (and universal) physical relation between the disk, emitting the primary radiation, and the hot electron corona emitting X-rays.
\end{abstract}

Key words: galaxies: active - galaxies: evolution - methods: statistical - quasars: general

Supporting material: machine-readable tables

\section{INTRODUCTION}

The distribution of X-ray and optical-UV properties in quasars, and their possible dependencies upon redshift, have been the subject of active investigations for more than 30 years (Tananbaum et al. 1979).

Such studies usually parametrized the relation between the $\mathrm{X}$-ray and optical-UV emission as a dependence between the logarithm of the monochromatic luminosity at $2500 \AA$, and the $\alpha_{\mathrm{ox}}$ parameter, defined as the slope of a power law connecting the monochromatic luminosity at $2 \mathrm{keV}$ and $L_{2500}: \alpha_{\mathrm{ox}}=$ $-0.384 \times \log \left[L_{2 \mathrm{keV}} / L_{2500}\right]$. A strong correlation between $\alpha_{\mathrm{ox}}$ and the optical luminosity at $2500 \AA$ is found, while $\alpha_{\text {ox }}$ is only marginally dependent upon redshift (but see Bechtold et al. 2003 for different results). The $\alpha_{\mathrm{ox}}$ distributions typically cover the range $1.2,1.8$, with a mean value of about 1.5 . A fairly significant correlation, albeit with a large scatter, is also found between $\alpha_{\mathrm{ox}}$ and the Eddington ratio ( $\lambda_{\text {edd }}$, Lusso et al. 2010, L10 hereafter; see also Vasudevan \& Fabian 2009).

The $\alpha_{\mathrm{ox}}-L_{2500}$ relation is the by-product of the non-linear correlation between $L_{2 \mathrm{keV}}$ and $L_{2500}\left(\log L_{2 \mathrm{keV}}=\right.$ $\gamma \log L_{2500}+\beta$ ) with a slope $\gamma$ of $0.5-0.7$ found in both optically and X-ray selected active galactic nucleus (AGN) samples (Vignali et al. 2003; Strateva et al. 2005; Steffen et al. 2006; Just et al. 2007; Lusso et al. 2010; Young et al. 2010). This implies that optically bright AGNs emit fewer X-rays (per unit UV luminosity) than optically faint AGNs (but see Yuan et al. 1998; La Franca et al. 1995 for a different interpretation).

Recently the nonlinear correlation between $L_{2 \mathrm{keV}}$ and $L_{2500}$ has been employed to reliably compute cosmological parameters such as $\Omega_{\mathrm{M}}$ and $\Omega_{\Lambda}$, and to build the first Hubble diagram for quasars which extends up to $z>6$ (Risaliti \& Lusso 2015), in excellent agreement with the analogous Hubble diagram for supernovae in the common redshift range (i.e., $z \sim 0.01-1.4$ ).

Understanding the $L_{2500}-L_{2} \mathrm{keV}$ relation thus provides a first hint about the nature of the energy generation mechanism in
AGNs, it is a first step towards understanding the structure of the AGN accretion disk and X-ray corona, and it can be used as a cosmological probe.

Yet, the dispersion along the $L_{2500}-L_{2 \mathrm{keV}}$ relation is found to be rather high, typically $\geqslant 0.35-0.4$ in $\log$ units, which may be presumably caused by the combination of multiple effects such as variability/not-simultaneous observations, poor optical-UV and X-ray data quality, and quasars (QSOs) which have intrinsically red continua and/or host galaxy contamination. All of these factors add noise to the correlation.

Here we analyze the $L_{2500}-L_{2} \mathrm{kev}$ relation using a sample of optically selected AGNs in the Sloan Digital Sky Survey seventh data release (Shen et al. 2011) with X-ray data from the latest release of the 3XMM serendipitous source catalog, 3XMM-DR5 (Rosen et al. 2015). Our main aim is to use this sample (much larger and more homogeneous than the previous ones in the literature) to understand the origin of the observed dispersion, and to evaluate the intrinsic dispersion of the $L_{2500}-L_{2 \mathrm{keV}}$ relation.

We adopt a concordance flat $\Lambda$-cosmology with $H_{0}=70 \mathrm{~km} \mathrm{~s}^{-1} \mathrm{Mpc}^{-1}, \Omega_{\mathrm{M}}=0.3$, and $\Omega_{\Lambda}=0.7$ (Komatsu et al. 2009).

\section{THE DATA}

Our sample starts with the catalog of quasar properties presented by Shen et al. (2011), which contains 105,783 spectroscopically confirmed broad-lined quasars. The SDSS quasar sample is cross-matched with the source catalog 3XMM-DR5 (Rosen et al. 2015). 3XMM-DR5 is the third generation catalog of serendipitous X-ray sources available online and contains 565,962 X-ray source detections (396,910 unique X-ray sources) made public on or before 2013 December $31 .{ }^{1}$ The net sky area covered (taking into account

\footnotetext{
1 http://xmmssc.irap.omp.eu/Catalogue/3XMM-DR5/3XMM_DR5.html
} 
overlaps between observations) is $\sim 877 \mathrm{deg}^{2}$, for a net exposure time $\geqslant 1 \mathrm{ks}$.

For the matching we have adopted a maximum separation of 3 arcsec to provide optical classification and spectroscopic redshift for all objects. ${ }^{2}$ This yields 4069 XMM observations (2605 unique sources ${ }^{3}, 601$ of which with multiple observations). The number of unique matches obtained after shifting all 3XMM-DR5 data by 1 arcmin in declination is zero, meaning that there are no spurious associations among the 2605 objects.

To define a reasonably "clean" sample we have applied the following quality cuts from the 3XMM-DR5 catalog: SUM_FLAG $<3$ (low level of spurious detections), and HIGH_BACKGROUND $=0$ (low background levels). ${ }^{4} \mathrm{We}$ have also excluded all QSOs in the SDSS-DR7 catalog flagged as broad absorption line (BAL), and radio emitters with radio loudness (flagged by R_6CM_2500A) higher than 10 (Kellermann et al. 1989). We have further neglected 3 quasars which were included in the BAL quasar catalog by Gibson et al. (2009), and the moderately radio-loud quasar J001115.23+144601.8, which was not flagged as such in the SDSS catalog. The remaining sample after these cuts is composed by 3304 XMM observations (2155 unique quasars, 470 of which with 2 or more observations). For sources with multiple observations (i.e., 1619 observations for the 470 quasars) we decided to take the one with the longest EPIC exposure. Our aim is to investigate to what degree the scatter on the $L_{2500}-L_{2 \mathrm{keV}}$ relation varies once the best possible sample of individual Xray detections is taken. The choice of the longest X-ray exposure is thus the most appropriate in order to minimize the possible "Eddington bias" due to the flux limit of each observation. We will further examine this point in Section 4.

We have estimated upper limits on the fluxes for the SDSS quasars that have been pointed by XMM-Newton but were not detected. We first matched the SDSS-DR7 quasar catalog with the list of 7781 observations included in the 3XMM-DR5 catalog finding 3481 objects within a circle of 15 arcmin (half of the field of view of the EPIC cameras). We then matched the 3481 quasars with the list of $2605 \mathrm{X}$-ray detected sources finding 2511 matches. This yields a sample of 970 SDSS quasars $(22 \%, 970 / 3481)$ which are in at least one XMM pointing but without a detection. Not all the X-ray detected quasars are retrieved with our the adopted circle of $30 \mathrm{arcmin}$ diameter (i.e., $2511 / 2605 \sim 95 \%$ ). This is due to the shape of the field of view of the two EPIC cameras that is not circular, meaning that there are regions where quasars are actually pointed, but lie at the edges of the field of view outside our adopted matching area. Additionally, the PN camera is shifted by 2 arcmin with respect to the MOS detectors (centered on the respective telescope optical axes), making the whole field of view more like an ellipses. ${ }^{5}$

We then use FLIX to compute robust $5 \sigma$ (corresponding to a likelihood threshold of 15.1) left censored data points ("upper

\footnotetext{
2 This value is rather conservative. The lists of counterparts with matching radii of $2.146,3.035$, and 3.439 are $90 \%, 99 \%$, and $99.73 \%$ complete, respectively (Watson et al. 2009).

3 Among these sources, 57 have multiple detections in the 3XMM-DR5 source catalog, but only one is within 3 arcsec.

4 For more details the reader should refer to the $3 \mathrm{XMM}$ catalog user guide at the following website: http://xmmssc.irap.omp.eu/Catalogue/3XMM-DR5/ 3XMM-DR5_Catalogue_User_Guide.html.

5 To recover the whole X-ray quasar sample of 2605 quasars we should use something like 17 arcmin. With such radius, we find that the number of undetected quasars is almost $45 \%$. This is clearly an upper limit on the number of undetected objects since many of them are outside the $X M M$ field of view.
}

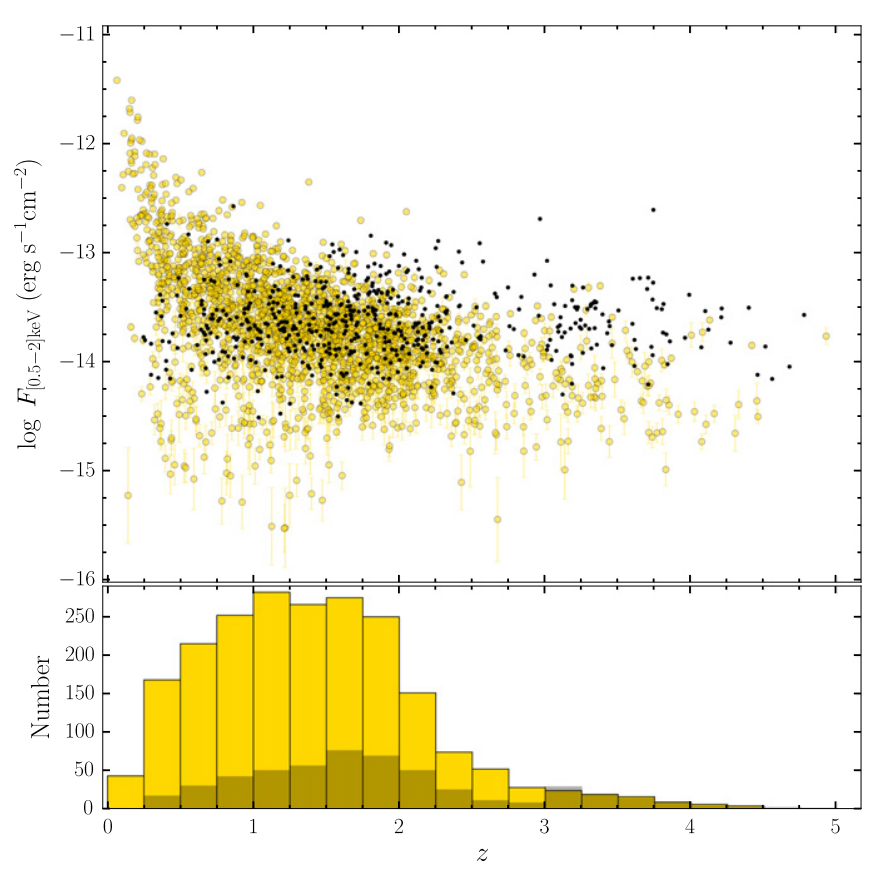

Figure 1. Top panel: distribution of the observed [0.5-2] keV flux vs. redshift for the main quasar sample. Upper limits are shown as small black points. Bottom panel: redshift distribution for the main quasar sample (yellow histogram) and for censored data (light black histogram).

limits"). Radio loud and BAL quasars are neglected following the same approach as above. This yields 789 SDSS quasars. For 254 quasars FLIX did not find any X-ray match in the soft band, and thus soft X-ray flux values were not available. This leads to a final sample of 535 quasars with X-ray upper limits.

To perform our analysis we utilized the observed continuum flux density values at rest-frame $2500 \AA\left(F_{2500}\right)$ as compiled by Shen et al. (2011), which take into account emission line contribution. $^{6}$ The interested reader should refer to their Section 3 for details on their spectral fitting procedure. Five quasars ( 2 objects with X-ray detection and 3 upper limits) do not have the $F_{2500}$ information and have been then excluded. Since uncertainties on $F_{2500}$ were not provided, we assumed a $2 \%$ uncertainty on the continuum flux measurement. The average uncertainty on the bolometric luminosity $\left(L_{\mathrm{bol}}\right)$ estimates in the SDSS quasar catalog is $\sim 3 \%$. Given that the bolometric measurements have an additional uncertainty due to the bolometric correction employed, we considered $2 \%$ a reasonable value for the uncertainties on continuum fluxes.

The main sample considered in the following analysis is shown in Figure 1 and it is composed by 2685 quasars (2153 $\mathrm{X}$-ray detections and 532 upper limits) spanning a redshift range of $0.065-4.925$.

The relevant source properties of the main X-ray detected and censored quasar samples are reported in Tables 1 and 2, respectively.

\subsection{X-Ray Luminosities}

To compute the rest-frame monochromatic luminosities at $2 \mathrm{keV}$ we first estimated the fluxes in the soft $(0.5-2 \mathrm{keV})$ and hard $(2-12 \mathrm{keV})$ energy bands as the sum of the EPIC fluxes listed in the 3XMM-DR5 catalog in bands 2 and 3, and bands 4 and 5, respectively. Uncertainties on these fluxes are computed

\footnotetext{
$\overline{6}$ These observed flux densities are divided by $(1+z)$ to shift these values into the rest-frame.
} 
Table 1

Optical and X-Ray Properties of the Main X-Ray Detected Quasar Sample

\begin{tabular}{|c|c|c|c|c|c|c|c|c|c|c|}
\hline SDSS Name & $\begin{array}{l}\text { R.A. } \\
\text { J2000.0 }\end{array}$ & $\begin{array}{c}\text { Decl. } \\
\text { J2000.0 }\end{array}$ & $z^{\mathrm{a}}$ & $\log L_{2500}{ }^{\mathrm{b}}$ & $\log L_{2 \mathrm{keV}^{\mathrm{c}}}^{\mathrm{c}}$ & DETID $^{d}$ & $\Gamma_{1}^{\mathrm{e}}$ & $\Gamma_{2}^{\mathrm{f}}$ & $\Gamma_{X}{ }^{g}$ & $\mathrm{~S} / \mathrm{N}^{\mathrm{h}}$ \\
\hline $000355.49+000736.4$ & 0.98121 & 0.126804 & 1.028 & 29.98 & $26.46 \pm 0.03$ & 103057510010006 & 0.78 & 0.62 & 1.67 & 16.90 \\
\hline $000439.97-000146.4$ & 1.16656 & -0.029582 & 0.583 & 29.23 & $25.02 \pm 0.12$ & 103057510010044 & -0.66 & -1.05 & 2.17 & 3.98 \\
\hline $000456.17+000645.5$ & 1.23405 & 0.112644 & 1.040 & 29.87 & $26.60 \pm 0.03$ & 103057510010004 & 1.05 & -0.26 & 1.53 & 19.09 \\
\hline
\end{tabular}

Notes.

${ }^{a}$ Spectroscopic redshifts from the SDSS-DR7 quasar catalog (Z_HW, improved redshifts from Hewett \& Wild 2010).

b Monochromatic $2500 \AA$ luminosities $\left(\mathrm{erg} \mathrm{s}^{-1} \mathrm{~Hz}^{-1}\right.$ ) from the SDSS-DR7 quasar catalog.

${ }^{\mathrm{c}}$ Monochromatic $2 \mathrm{keV}$ luminosities $\left(\mathrm{erg} \mathrm{s}^{-1} \mathrm{~Hz}^{-1}\right.$ ) of the 1 st $X M M$ longest exposure.

${ }^{\mathrm{d}}$ Number which identifies each entry (unique to each X-ray detection) in the 3XMM-DR5 catalog.

e Optical (0.3-1 micron) SED power-law fit slope.

${ }^{\text {f }}$ UV (1450-3000 A) SED power-law fit slope.

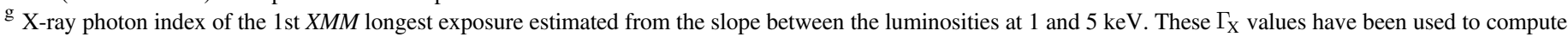
$L_{2 \mathrm{keV}}$ and to select the clean quasar sample.

${ }^{\mathrm{h}}$ X-ray signal-to-noise of the 1st $X M M$ longest exposure: S/N $=$ EP_8_CTS/EP_8_CTS_ERR.

(This table is available in its entirety in machine-readable form.)

Table 2

Optical and X-Ray Properties of the Main X-Ray Censored Quasar Sample

\begin{tabular}{lccccrrr}
\hline \hline SDSS Name & R.A. & $\begin{array}{c}\text { Decl. } \\
\text { J2000.0 }\end{array}$ & $z^{\mathrm{a}}$ & $\log L_{2500}{ }^{\mathrm{b}}$ & $\log L_{2 \mathrm{keV}^{\mathrm{c}}}$ & $\Gamma_{1}^{\mathrm{d}}$ & $\Gamma_{2}^{\mathrm{e}}$ \\
\hline $000525.12+001745.2$ & 1.35469 & 0.295899 & 1.030 & 30.13 & $26.13 \pm 0.08$ & 1.29 \\
$001030.55+010006.0$ & 2.62731 & 1.00168 & 0.378 & 29.38 & $25.11 \pm 0.06$ & 0.33 \\
$001201.87+005259.7$ & 3.00782 & 0.883254 & 1.637 & 30.29 & $26.51 \pm 0.10$ & 0.60 \\
\hline
\end{tabular}

Notes.

${ }^{\text {a }}$ Spectroscopic redshifts from the SDSS-DR7 quasar catalog (Z_HW, improved redshifts from Hewett \& Wild 2010).

${ }^{\mathrm{b}}$ Monochromatic $2500 \AA$ A luminosities $\left(\mathrm{erg} \mathrm{s}^{-1} \mathrm{~Hz}^{-1}\right.$ ) from the SDSS-DR7 quasar catalog.

${ }^{\mathrm{c}}$ Monochromatic $2 \mathrm{keV}$ luminosities (erg s${ }^{-1} \mathrm{~Hz}^{-1}$ ) of the $1 \mathrm{st} X M M$ longest exposure.

${ }^{\mathrm{d}}$ Optical (0.3-1 micron) SED power-law fit slope.

${ }^{\mathrm{e}} \mathrm{UV}(1450-3000 \AA)$ SED power-law fit slope.

(This table is available in its entirety in machine-readable form.)

by summing in quadrature the cataloged flux uncertainties in bands 2 and 3 and in bands 4 and 5 for the soft and the hard band, respectively. A power-law spectral model with a photon index, $\Gamma_{\mathrm{X}}=1.7$ and a hydrogen absorbing column density of $N_{\mathrm{H}}=3 \times 10^{20} \mathrm{~cm}^{-2}$ was assumed to convert count rates into fluxes (Rosen et al. 2015).

To plot the X-ray information in the $\log \nu-\log \nu L(\nu)$ restframe plane we have first estimated the luminosity at the geometric mean of the soft $(1 \mathrm{keV})$ and hard $(5 \mathrm{keV})$ energy bands by assuming an average photon index of 1.7 in both bands. Luminosities are then blueshifted to the rest-frame. The rest-frame monochromatic luminosities at $2 \mathrm{keV}$ is finally obtained by interpolation if the source redshift is lower than 1, by extrapolation considering the slope between the luminosities described above for higher redshifts. Uncertainties on monochromatic luminosities $\left(L_{\nu} \propto \nu^{-\gamma}\right)$ from interpolation (extrapolation) between two values $L_{1}$ and $L_{2}$ are computed as

$$
\delta L=\sqrt{\left(\frac{\partial L}{\partial L_{1}}\right)^{2}\left(\delta L_{1}\right)^{2}+\left(\frac{\partial L}{\partial L_{2}}\right)^{2}\left(\delta L_{2}\right)^{2}} .
$$

For X-ray undetected quasars we have estimated the EPIC fluxes in bands 2 and 3 as the mean of the upper limit flux values in all cameras. The soft $(0.5-2 \mathrm{keV})$ flux is then the sum of bands 2 and 3

$$
F_{[0.5-2] \mathrm{keV}, \mathrm{ul}}=\frac{\sum_{b} b \_2 \_F U P L}{n}+\frac{\sum_{b} b \_3 \_F U P L}{n},
$$

where $b=\operatorname{MOS} 1, \operatorname{MOS} 2$, and pn and $n$ is the number of cameras with non-zero flux value. Monochromatic $2 \mathrm{keV}$ luminosities have been computed using a photon index of 1.7.

For each detected object we have also computed the EPIC sensitivity ( $5 \sigma$ minimum detectable flux) at $2 \mathrm{keV}$. To do that, we have considered the pn, MOS1, and MOS2 on-time ${ }^{7}$ and off-axis values, where both MOS1 and MOS2 on-time and off-axis have been combined. The total MOS on-time and off-axis are the largest and smaller values of the two individual cameras, respectively. We then estimated the minimum detectable flux in the soft band as a function of the exposure time following the relations plotted in Figure 3 by Watson et al. (2001) for both pn and MOS. We then corrected this sensitivity for the pn and MOS vignetting factor as a function of their respective off-axis values. The same vignetting correction for both pn and MOS has been considered. The sensitivity flux values at $2 \mathrm{keV}\left(F_{\min }\right)$ are then estimated assuming a photon index of 1.7 and finally combined. We have taken the sum of the pn and MOS fluxes in the case where both values are available.

\footnotetext{
7 The total good exposure time (in seconds) of the CCD where the source is detected.
} 


\section{STATISTICAL ANALYSIS}

Previous studies on optically selected AGNs reported a relationship between $L_{2 \mathrm{keV}}$ and $L_{2500}$ in the form $L_{2 \mathrm{keV}} \propto L_{2500}^{\gamma}$. The best-fit values of the exponent are between $0.7 \div 0.8$ (Avni \& Tananbaum 1982; Chanan 1983; Kriss \& Canizares 1985; Wilkes et al. 1994; Yuan et al. 1998; Vignali et al. 2003; Strateva et al. 2005; Steffen et al. 2006; Just et al. 2007; Lusso et al. 2010; Young et al. 2010), estimated from an ordinary least-square (OLS) bisector analysis (Isobe et al. 1990). The bisector treats $X$ and $Y$ variables symmetrically, and it has been usually justified by the fact that (1) the choice of the independent variable (between $L_{2500}$ and $L_{2 \mathrm{keV}}$ ) was not straightforward, and (2) methods which minimize residuals of the dependent variable are subject to effects caused by the large observed luminosity dispersion (i.e., 0.35-0.4 dex, see Tang et al. 2007).

The observed $L_{2500}-L_{2 \mathrm{keV}}$ relation (or its by-product: the $\alpha_{\mathrm{ox}}-L_{2500}$ relation) provides insights into the radiation mechanism in quasars. The nonlinearity of such correlation implies that more optically luminous AGNs emit less X-rays per unit of UV luminosity than less luminous AGNs. The X-ray properties in quasars have been attributed to the presence of a plasma of relativistic electrons at high temperatures ( $T \sim 10^{9} \mathrm{~K}$, the so-called corona) in the vicinity of the accreting supermassive black hole. Optical-UV photons from the accretion disk (parametrized by $L_{2500}$ ) are Compton upscattered by hot electrons and lead to the formation of a power law spectrum in the X-rays (parametrized by $L_{2} \mathrm{keV}$ ) accompanied by a high energy cut-off at the electrons' temperature (Haardt \& Maraschi 1991, 1993; Dadina 2008). The observed correlation thus suggests that disk and corona are coupled, and that the corona parameters should be then dependent on the UV luminosity. In this framework, $L_{2500}$ and $L_{2 \mathrm{keV}}$ are not independent parameters, and therefore the regression methods to fit the data needs to be chosen carefully.

The situation is even more complex in the case of samples with censored data. We adopted the LINMIX_ERR ${ }^{8}$ method (Kelly 2007), which is argued to be among the most robust regression algorithms with the possibility of reliable estimation of intrinsic random scatter on the regression (f). LINMIX_ERR accounts for measurement uncertainties on both independent and dependent variable, nondetections, and intrinsic scatter by adopting a Bayesian approach to compute the posterior probability distribution of parameters, given observed data.

To investigate whether the fitting results depend on the adopted LINMIX_ERR method, we have also considered the Astronomy Survival Analysis software package (ASURV rev. 1.2; Isobe et al. 1990; Lavalley et al. 1992), which is widely used in the literature. ASURV implements the bivariate dataanalysis methods and also properly treats censored data using the survival analysis methods (Feigelson \& Nelson 1985; Isobe et al. 1986). We have employed the full parametric estimate and maximized (EM) regression algorithm to perform the linear regression of the data, and the semiparametric Buckley-James regression algorithm (Buckley \& James 1979). The EM regression algorithm is based on the OLS regression of the dependent variable $Y$ against the independent variable $X$ (OLS $[Y \mid X])$. The regression line is defined in such a way that it minimizes the sum of the squares of the $Y$ residuals. However, this regression method is less powerful than LINMIX_ERR

\footnotetext{
8 This algorithm has been implemented in Python and its description can be found at http://linmix.readthedocs.org/en/latest/src/linmix.html.
}

since it does not account for measurement uncertainties, and it does not provide an estimate of the intrinsic scatter. For censored data, we will present our findings in Section 3.1 from both the LINMIX_ERR and EM regression methods, although the latter is reported just for comparison with previous works in the literature (in all cases the results from the Buckley-James regression algorithm agreed with EM within the uncertainties).

Censored sample are likely to be unbiased, but the analysis of the scatter along the $L_{2500}-L_{2 \mathrm{kev}}$ relationship may not be straightforward, since it strongly depends on the weights assumed in the fitting algorithm. Additionally, there is often the situation in surveys where upper limits are not provided. In the case of flux-(or magnitude) limited surveys, objects with an expected luminosity (based on the observed $L_{2500}-L_{2 \mathrm{keV}}$ relation) near to the sample flux limit will be observed only in case of positive fluctuations. Considering only detections may thus introduce a bias in the $L_{2500}-L_{2 \mathrm{keV}}$ relationship, and this should be more relevant in the $\mathrm{X}$-rays, since the relative flux/luminosity interval is much smaller than in the opticalUV. Therefore, one needs to find an alternative method to obtain a sample where biases are minimized even without the inclusion of censored data. One possibility is to include only objects that would be observed even in case of negative flux fluctuations. We explored the flux-limit bias in the X-ray detected quasar sample in Appendix, where we prove that this bias is not significantly affecting our main results. We have thus examined the correlation between $L_{2500}$ and $L_{2 \mathrm{keV}}$ where nondetections are neglected.

To this goal, we employed an orthogonal distance regression (ODR) fitting procedure in addition to the LINMIX_ERR algorithm. The ODR regression treats $X$ and $Y$ variables symmetrically and minimizes both the sum of the squares of the $X$ and $Y$ residuals. ${ }^{9}$ We note that the LINMIX_ERR and ODR algorithms are mathematically different and, in principle, should not be used interchangeably. However, the use of multiple fitting methods, although distinct, is still useful, especially in the case of large scatter.

\subsection{The $L_{2500}-L_{2 \mathrm{keV}}$ Relation: Censored Data}

We computed slope and intercept of the $L_{2500}-L_{2} \mathrm{kev}$ relation for the main sample and we investigated how the fit parameters vary depending on possible selection criteria. The findings from the EM regression and the LINMIX_ERR algorithms are summarized in Table $3 .{ }^{10}$

Comparing our best-fit parameters with those obtained from optically selected samples, we find that our slope is fully consistent within $1 \sigma$ with the results presented by S06 $\left(\beta_{\mathrm{S} 06}=0.642 \pm 0.021\right)$ and $\mathrm{J} 07\left(\beta_{\mathrm{J} 07}=0.636 \pm 0.018\right)$, and with the ones by $\operatorname{L10}\left(\beta_{\mathrm{L} 10}=0.599 \pm 0.027\right)$ from $\operatorname{OLS}(Y \mid X)$.

We note that continuum flux measurements in the Shen et al. catalog were neither corrected for intrinsic extinction/reddening, nor for host contamination. Therefore, we singled out a sub-sample of objects where both reddening and host contaminations are reduced at minimum. To minimize hostgalaxy and reddening contamination we followed a similar approach as in Risaliti \& Lusso (2015). We computed for each object the slope $\Gamma_{1}$ of a $\log (\nu)-\log (\nu L \nu)$ power law in the $0.3-1 \mu \mathrm{m}$ (rest frame) range, and the analogous slope $\Gamma_{2}$ in the

\footnotetext{
9 http://docs.scipy.org/doc/scipy/reference/odr.html

${ }^{10}$ We considered the dispersion value output of the EM regression algorithm as representative of the scatter along the correlation including censored data.
} 
Table 3

Results from Correlations Analysis of Censored Data

\begin{tabular}{|c|c|c|c|c|c|c|c|c|}
\hline Sample & $\gamma$ & $\begin{array}{c}\beta \\
\text { LINMIX_ERR }\end{array}$ & $f$ & $\gamma$ & EM & $\sigma$ & $N_{\mathrm{ul}}$ & $N_{\text {tot }}$ \\
\hline Main & $0.582 \pm 0.014$ & $8.664_{-0.417}^{+0.403}$ & $0.162 \pm 0.005$ & $0.586 \pm 0.014$ & $8.523 \pm 0.428$ & 0.43 & 532 & 268 \\
\hline$E(B-V) \leqslant 0.1$ & $0.592 \pm 0.015$ & $8.345_{-0.461}^{+0.471}$ & $0.150 \pm 0.005$ & $0.596 \pm 0.016$ & $8.237 \pm 0.492$ & 0.41 & 421 & 2319 \\
\hline$E(B-V) \leqslant 0.1-\mathrm{S} / \mathrm{N}>3$ & $0.593 \pm 0.016$ & $8.334_{-0.482}^{+0.472}$ & $0.150 \pm 0.005$ & $0.596 \pm 0.016$ & $8.214 \pm 0.494$ & 0.42 & 430 & 2319 \\
\hline$E(B-V) \leqslant 0.1-\mathrm{S} / \mathrm{N}>5$ & $0.583 \pm 0.017$ & $8.585_{-0.519}^{+0.518}$ & $0.173 \pm 0.004$ & $0.584 \pm 0.018$ & $8.562 \pm 0.537$ & 0.45 & 635 & 231 \\
\hline$E(B-V) \leqslant 0.1-\mathrm{S} / \mathrm{N}>5-1.6 \leqslant \Gamma_{\mathrm{X}} \leqslant 2.8$ & $0.584 \pm 0.015$ & $8.627_{-0.462}^{+0.468}$ & $0.113 \pm 0.005$ & $0.583 \pm 0.016$ & $8.658 \pm 0.476$ & 0.35 & 527 & 182 \\
\hline
\end{tabular}

1450-3000 $\AA$ range (rest frame). The $\Gamma_{1}-\Gamma_{2}$ distribution is shown in Figure 2. We selected all sources with $\Gamma_{1}-\Gamma_{2}$ centered at $E(B-V)=0.0$ with a radius of 1.1 , which roughly corresponds to $E(B-V) \simeq 0.1$. We found 2319 quasars that matched this criteria (421 upper limits).

To avoid large uncertainties on the X-ray flux measurements due to unreliable source counts, we have considered as upper limits all X-ray detected quasars with a ratio between the EPIC source count in the $0.2-12.0 \mathrm{keV}$ band and its uncertainty lower than $3\left(\mathrm{~S} / \mathrm{N}=\mathrm{EP} \_8 \_C T S / E P \_8 \_C T S \_E R R ~ \leqslant 3\right.$, i.e., we have considered X-ray detected all objects with at least 3 sigma counts measurement). We also examined a higher threshold of $\mathrm{S} / \mathrm{N}=5$.

Since soft X-ray fluxes may contain some level of absorption, we included only X-ray detected quasars with a photon index $\Gamma_{\mathrm{X}}$ in the range 1.6-2.8, which roughly corresponds to an average $\Gamma_{\mathrm{X}} \sim 2$ with a dispersion of 0.3 . Our "photometric" $\Gamma_{X}$ values represent the slope between the 1 and $5 \mathrm{keV}$ luminosities and, although they cannot be considered as reliable as the spectroscopic measurements, are reasonable tracers of X-ray absorption. To further minimize the level of X-ray absorption, we have also studied a narrower $\Gamma_{\mathrm{X}}$ interval of 1.9-2.8 $\left(\left\langle\Gamma_{\mathrm{X}}\right\rangle \sim 2.1\right.$ with a dispersion of 0.2 , consistent with Young et al. 2009). Given the observed $\Gamma_{X}$ range (up to 2.8), some soft-excess contribution for low-Z QSOs might be still present. We have thus repeated the analysis further reducing the $\Gamma_{\mathrm{X}}$ range (up to 2.4), but, besides loosing statistics, our results are not affected.

The conditions listed above have the only aim of selecting a sample of quasars with homogeneous SEDs and to minimize the number of red/reddened quasars in both optical and X-ray bands. These requirements yield a sample of 1228 quasars, 485 of which are upper limits. Fit parameters for such a sample (slope, intercept, and dispersion) are fully consistent with previous estimates in the literature. Our exploration of the parameter space shows that the dispersion of the $L_{2500}-L_{2} \mathrm{keV}$ relation is mainly driven by poor X-ray data, X-ray absorption, and quasars with red continua and/or host galaxy contamination for which the optical flux measurement cannot be properly recovered.

\subsection{The $L_{2500}-L_{2 \mathrm{kev}}$ Relation: $X$-Ray Detected Data}

We then repeated the analysis in Section 3.1 only for the sample of X-ray detected quasars where we applied the same series of filters as the ones already discussed. The results are plotted in the bottom panel of Figure 3, while the findings from the regression algorithms for the different selection criteria are presented in Table 4. The dispersion on this final sample of Xray detected quasars reduces from $\sim 0.45$ to 0.24 dex by applying the same set of filters as the ones of the censored data,

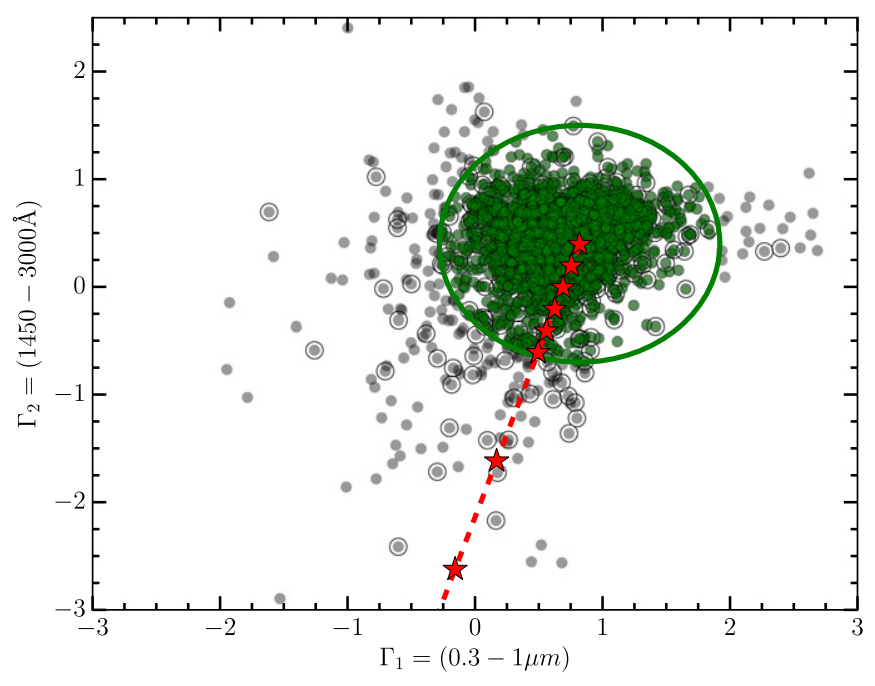

Figure 2. Distribution of the whole quasar sample (excluding radio-loud and BAL sources) in a $\Gamma_{1}-\Gamma_{2}$ plot, where $\Gamma_{1}$ and $\Gamma_{2}$ are the slopes of a power law in the $\log \nu-\log \left(\nu L_{\nu}\right)$ plane, at $0.3-1 \mu \mathrm{m}$ and $1450-3000 \AA$, respectively. The dashed red line is obtained by assuming increasing dust extinction following the extinction law of Prevot et al. (1984) for the quasar SED estimated by Richards et al. (2006). The red stars represent the value of $\Gamma_{1}-\Gamma_{2}$ for $E(B-V)=(0.0,0.02,0.04,0.06,0.08,0.1,0.2,0.3)$. The green circle is centered at $E(B-V)=0.0$ with a radius of $\sim 1$ (i.e., $E(B-V) \simeq 0.1$ ). Sources within the circle are highlighted in green. Open circles highlight X-ray non-detections.

which is significantly lower than what previously reported in literature (i.e., $>0.35$ Vignali et al. 2003; Strateva et al. 2005; Steffen et al. 2006; Just et al. 2007; Lusso et al. 2010; Young et al. 2010). Additionally, there is no significant variation on both slope and intercept (within their uncertainties) among the different selections, with the slope being rather constant around $0.6-0.65$. The results from the ODR fitting procedure seems to show slightly steeper slopes than LINMIX_ERR, although the disagreement is below $2 \sigma$.

We stress that our selection criteria are extremely simple and they have the only aim to favor blue quasars having homogeneous SED, where we can robustly estimate both $L_{2500}$ and $L_{2 \mathrm{keV}}$ with minimum contamination from hostgalaxy/reddening, and absorption in X-rays. The observed slope and intercept estimated for this sample can be considered representative of intrinsic values of the observed $L_{2500}-L_{2} \mathrm{keV}$ relation. The fact that the observed $L_{2500}-L_{2 \mathrm{keV}}$ relation is very tight is the manifestation of a common physical nature in quasars. Yet, at present, the details on the physics governing the interplay between the $\mathrm{X}$-ray corona and the accretion disk is still not well understood. 


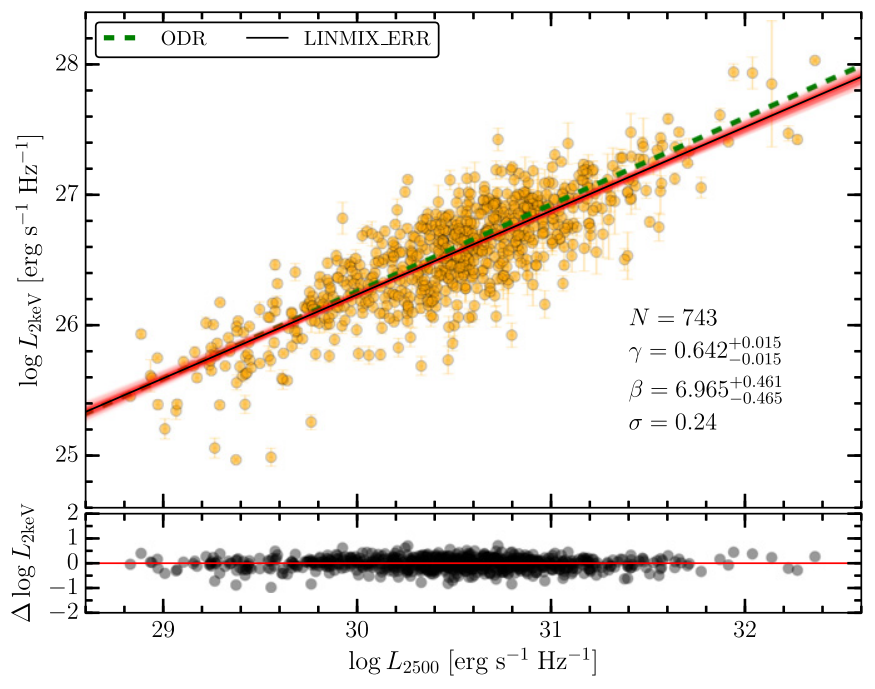

Figure 3. Rest-frame monochromatic luminosities $\log L_{2 \mathrm{keV}}$ against $\log L_{2500}$ for the X-ray detected (orange circles) quasar samples as described in Section 3.2. The results from the ODR regression (dashed line) and the LINMIX_ERR (thin solid line) for the selected sample are also reported. Red thin lines represent 400 different realizations of the $L_{2500}-L_{2 \mathrm{keV}}$ relation from LINMIX_ERR. The LINMIX_ERR regression results for the dispersion, slope, and intercept (with their uncertainties) are also reported. The lower panel shows the residuals of $\log L_{2} \mathrm{keV}$ and $\log L_{2500}$ with respect to the LINMIX_ERR bestfit line.

\section{X-RAY VARIABILITY}

One of the contributors to the scatter in the $L_{2500}-L_{2 \mathrm{keV}}$ relationship can be the X-ray emission variation among different observations. To quantify the extent of such variability, we have compared the monochromatic $2 \mathrm{keV}$ luminosities of the 2nd $X M M$ longest exposure to the ones of the longest $X M M$ exposure. We have considered the 470 quasars with more than two $X M M$ observations and we have applied the same selection criteria as the ones discussed in Section 3.1, leading to a sample 159 quasars. We have then estimated $L_{2} \mathrm{keV}$ for the second longest exposure following the same approach as for the longest one. The comparison between these two luminosities is shown in Figure 4. The dispersion along the one-to-one relation (red dashed line) is 0.21 dex. The procedure to convert counts into fluxes/luminosities introduces additional scatter, which is not due to X-ray variability alone. In fact, the observed dispersion between $L_{2 \mathrm{keV}}(1 \mathrm{st})$ and $L_{2 \mathrm{keV}}$ (2nd) also depends on the deviation between the offaxis angles of the two observations, with the minimum difference (i.e., offaxis $(1 \mathrm{st})$-offaxis $(2 \mathrm{nd})=0.02)$ having the lowest dispersion $(\sim 0.17 \mathrm{dex})$. The majority of the sources with multiple, almost on-axis, observations are indeed pointed objects where uncertainties due to flux calibration, background subtraction, and vignetting correction are almost negligible. We find that the dispersion due to X-ray variability, bad calibrations, etc., is thus on the order of $\sim 0.12$ dex.

To provide another quantitative measure of the amplitude of $\mathrm{X}$-ray variability in our sample we followed a similar procedure as the one described by Gibson \& Brandt (2012). We computed the fractional variation $\left(F_{\mathrm{var}}\right)$ as

$$
F_{\mathrm{var}} \equiv\left(c_{i}-c_{j}\right) /\left(c_{i}+c_{j}\right),
$$

where $c_{i}$ and $c_{j}$ are the count rates for the 1st and 2nd XMM longest exposure, respectively. Each measurement of $F_{\mathrm{var}}$ between the two exposures is associated with a rest-frame time measurement $\Delta t_{\mathrm{sys}}$ defined as $\Delta t_{\mathrm{sys}}=\left(t_{j}-t_{i}\right) /(1+z)$, where we have taken the absolute value of $\left(t_{j}-t_{i}\right)$. We then assumed an intrinsic Gaussian distribution for $F_{\mathrm{var}}$ and estimated the standard deviation of this Gaussian distribution $\left(\sigma\left(F_{\text {var }}\right)\right)$ using the likelihood method described by Maccacaro et al. (1988). We then binned $\Delta t_{\text {sys }}$ in three intervals of about 50 epochs each. The upper and lower panel of Figure 5 shows our results for $F_{\text {var }}$ and $\sigma\left(F_{\mathrm{var}}\right)$ as a function of $\Delta t_{\mathrm{sys}}$ for each quasar epoch, respectively. Each value of $\sigma\left(F_{\mathrm{var}}\right)$ is plotted at the median $\Delta t_{\text {sys }}$ in the considered bin. The dashed line represents a linear fit of $\sigma\left(F_{\mathrm{var}}\right)$, which is parametrized as

$$
\sigma\left(F_{\mathrm{var}}\right)=(0.066 \pm 0.033) \log \Delta t_{\mathrm{sys}}+(-0.200 \pm 0.215)
$$

The level of fractional variation is $\sim 20 \%$, which corresponds to a dispersion of $\sim 0.08 \mathrm{dex}$ in $\log$. If we consider timescales longer than one week $\left(\Delta t_{\mathrm{sys}} \geqslant 6 \times 10^{5} \mathrm{~s}\right)$, given that such timescales represent the majority of our data set, we have that the amplitude of fractional variation is $\sim 31 \%$ (i.e., $\sim 0.12 \mathrm{dex}$ ). Unfortunately, we do not have enough data to provide significant constraints on $\sigma\left(F_{\mathrm{var}}\right)$ as a function of $\Delta t_{\text {sys }}$, hence we will consider the latter value as more representative of our sample. This value is also in agreement with the one we have estimated from the analysis of the off-axis discussed above.

As a comparison, Gibson \& Brandt (2012) presented a detailed analysis of the quasar X-ray variability as a function of timescale, redshift, luminosity, and optical spectral properties. Their sample consists of 264 optically selected quasars from SDSS-DR5 with at least two X-ray observations (three or more are available for 82 quasars) from the Chandra public archive. They found that $\sigma\left(F_{\mathrm{var}}\right)$ is $\sim 16 \%-17 \%$ (corresponding to a dispersion of $\sim 0.065 \mathrm{dex}$ in $\log$ ) at $\Delta t_{\text {sys }}>5 \times 10^{5} \mathrm{~s}$, which is a rather lower value with respect to our. This may be partly due to the combination of higher statistics and the lower background level of their Chandra data.

Lanzuisi et al. (2014) have analyzed a sample of 638 AGNs (340 Type 1) with XMM-Newton observations in the COSMOS field over 3.5 year to study their long term variability. The amplitude of their fractional variation is on the order of $\sim 30 \%$, in close agreement with our findings.

For the sample of 159 quasars with multiple observations, we have then calculated $\gamma$ and $\beta$ of the $L_{2500}-L_{2 \mathrm{keV}}$ relation finding $\gamma=0.672 \pm 0.035, \beta=6.044_{-1.075}^{+1.166}$, and a dispersion of 0.23 dex in agreement with the best selected quasar sample. If we instead consider the $L_{2} \mathrm{kev}$ values estimated as the average between the 1st and 2nd XMM longest exposure, the dispersion on the $L_{2500}-L_{2} \mathrm{keV}$ relation reduces to 0.21 dex (see Figure 6), while both slope and intercept are still in agreement. Results are not affected even considering $L_{2 \mathrm{keV}}$ values estimated as the average of all $X M M$ observations. This is likely because the majority of these objects have two observations $(55 \%, 88 / 159)$, with solely 35 quasars with more than 3 detections.

Summarizing, we found that the amplitude of X-ray variability in the sample of 159 quasars with multiple observations is around 0.12 dex.

\section{SIMULTANEOUS OBSERVATIONS}

To test the influence of non-simultaneous measurements on the $L_{2500}-L_{2 \mathrm{keV}}$ relationship we extracted simultaneous data 
Table 4

Results from Correlations Analysis of Detected Data

\begin{tabular}{|c|c|c|c|c|c|c|c|c|}
\hline Sample & $\gamma$ & $\begin{array}{c}\beta \\
\text { LINMIX_ERR }\end{array}$ & $f$ & $\sigma$ & $\gamma$ ODR & $\beta$ & $\sigma$ & $N_{\text {tot }}$ \\
\hline Main & $0.583 \pm 0.014$ & $8.697_{-0.412}^{+0.415}$ & $0.147 \pm 0.005$ & 0.42 & $0.593 \pm 0.010$ & $8.558 \pm 0.303$ & 0.45 & 2153 \\
\hline$E(B-V) \leqslant 0.1$ & $0.596 \pm 0.016$ & $8.279_{-0.478}^{+0.460}$ & $0.135 \pm 0.005$ & 0.40 & $0.618 \pm 0.011$ & $7.773 \pm 0.346$ & 0.44 & 1898 \\
\hline$E(B-V) \leqslant 0.1-\mathrm{S} / \mathrm{N}>3$ & $0.596 \pm 0.015$ & $8.279_{-0.469}^{+0.462}$ & $0.135 \pm 0.005$ & 0.40 & $0.618 \pm 0.011$ & $7.773 \pm 0.346$ & 0.44 & 188 \\
\hline$E(B-V) \leqslant 0.1-\mathrm{S} / \mathrm{N}>5$ & $0.589 \pm 0.015$ & $8.539_{-0.465}^{+0.456}$ & $0.113 \pm 0.004$ & 0.35 & $0.619 \pm 0.012$ & $7.752 \pm 0.363$ & 0.38 & 168 \\
\hline$E(B-V) \leqslant 0.1-\mathrm{S} / \mathrm{N}>5-1.6 \leqslant \Gamma_{\mathrm{X}} \leqslant 2.8$ & $0.596 \pm 0.014$ & $8.392_{-0.398}^{+0.415}$ & $0.074 \pm 0.003$ & 0.28 & $0.634 \pm 0.012$ & $7.332 \pm 0.365$ & 0.30 & 1298 \\
\hline
\end{tabular}
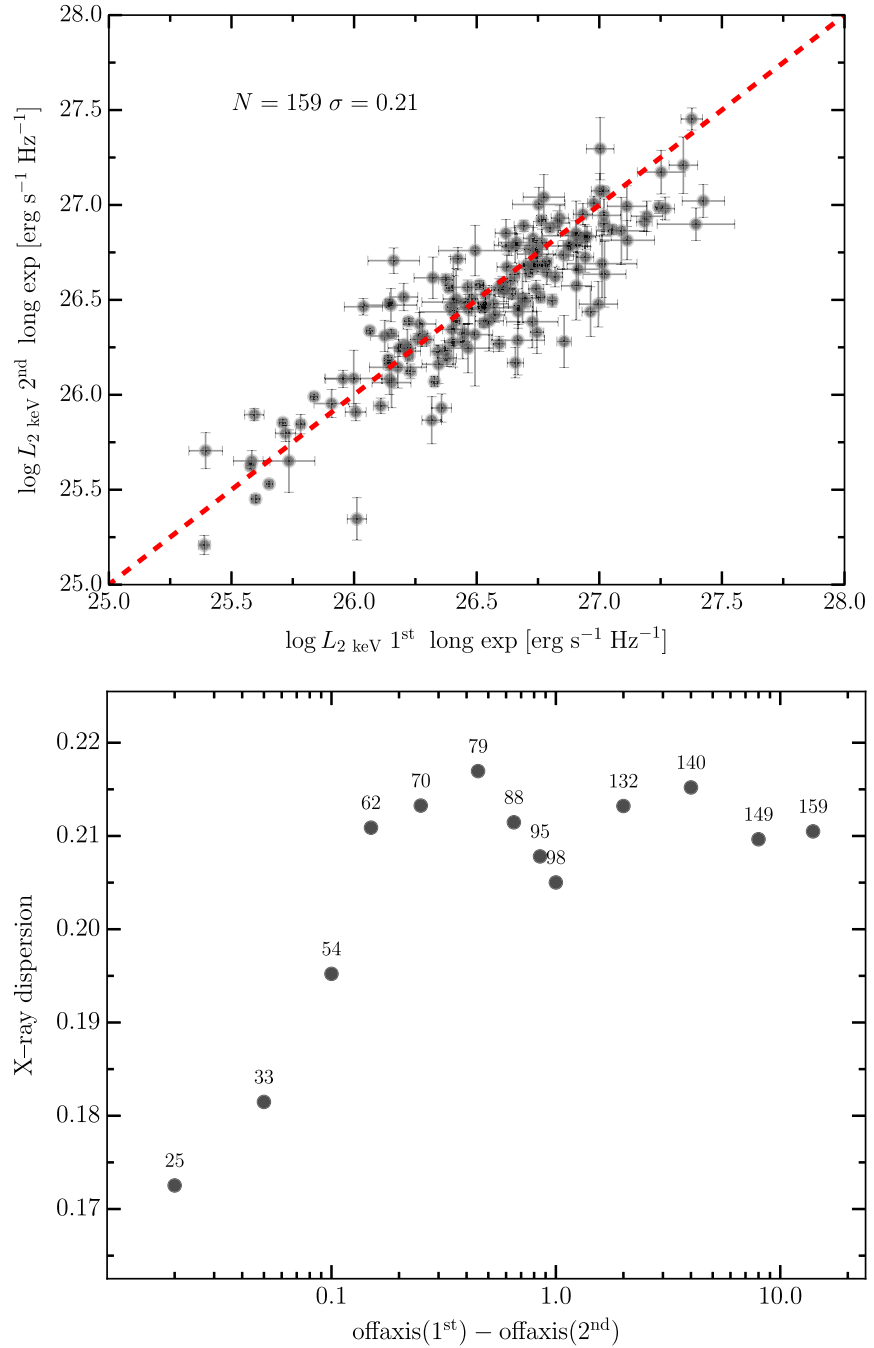

Figure 4. Upper panel: monochromatic $2 \mathrm{keV}$ luminosities of the 2nd $X M M$ longest exposure as a function of those with the longest $X M M$ exposure for the selected quasar sample with multiple observations. The dispersion along the one-to-one relation (red dashed line) is $0.21 \mathrm{dex}$ and it roughly quantifies the extent of X-ray variability on the $L_{2500}-L_{2 \mathrm{kev}}$ relationship. Lower panel: variability dispersion as a function of the offaxis difference between the two longest $X M M$ observations. The number of objects in each offaxis bin is reported on top of each point.

from the latest release of XMM-Newton Optical Monitor Serendipitous UV Source Survey catalog (Page et al. 2012, XMMSUSS2.1) available online. ${ }^{11}$ XMMSUSS2.1 includes all data to the end of 2013 and contains 7170 observations for a

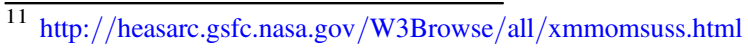
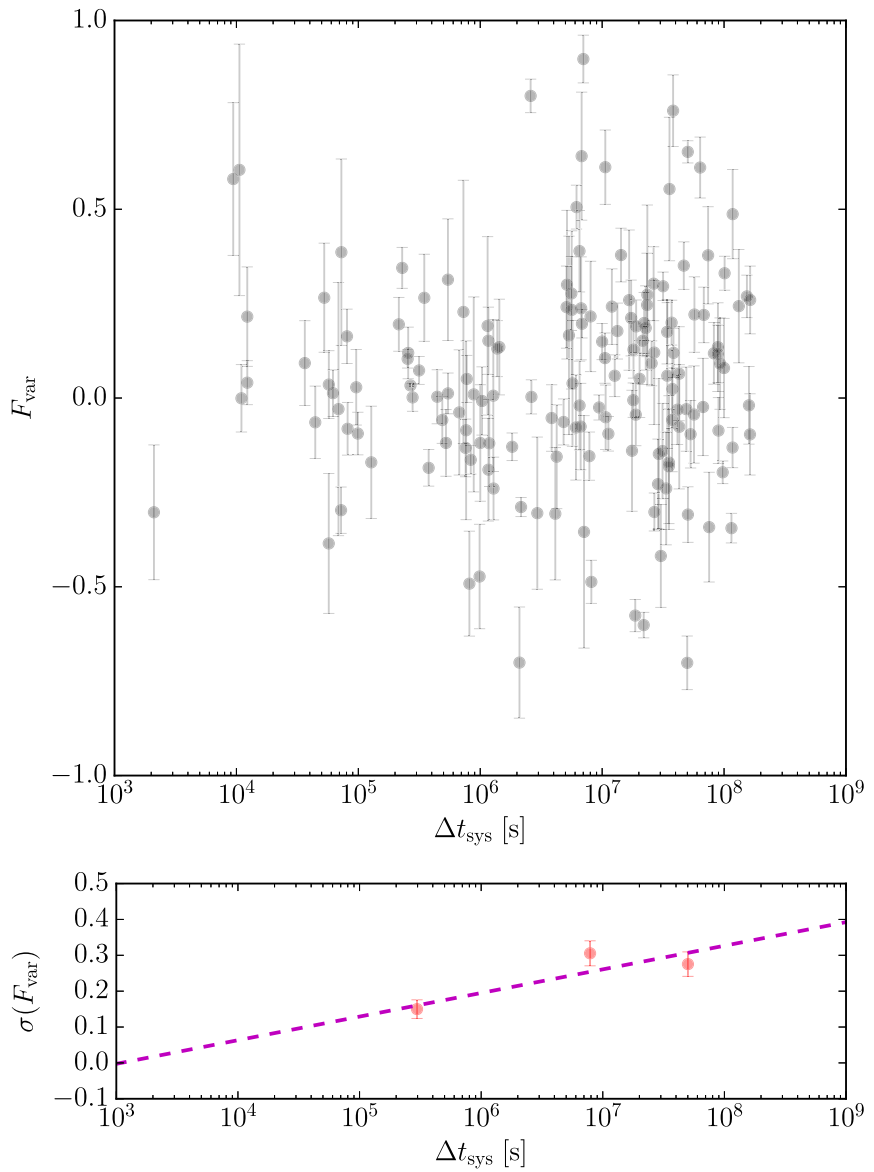

Figure 5. Upper panel: distribution of $F_{\mathrm{var}}$ as a function of the rest-frame time measurement $\Delta t_{\text {sys }}$. Each point is estimated taking the 1st and 2nd $X M M$ longest exposure. Lower panel: Gaussian dispersion of $F_{\mathrm{var}}$ as a function of the rest-frame time measurement $\Delta t_{\text {sys. }}$. The magenta dashed line is a linear fit of $\sigma\left(F_{\mathrm{var}}\right)$ (see the text for details).

total number of entries of 6,246,432 (4,329,363 unique sources) detected in one to six broad-band UV and optical filters in the Optical Monitor (XMM-OM) on board the XMMNewton observatory. We matched the sample of 2090 SDSS quasars with the XMMSUSS2.1 catalog within 3 arcsec and, by imposing the same XMM-Newton observation in the XMMSUSS2.1 catalog, we found 1043 entries (597 unique quasars). ${ }^{12}$

To obtain the rest-frame monochromatic luminosities at $2500 \AA$ we used all the available photometry compiled in the

${ }^{12}$ This has been done by comparing the OBS_ID and OBSID flags in the 3XMM-DR5 and XMMSUSS2.1 catalogs, which uniquely identify XMMNewton pointings. 


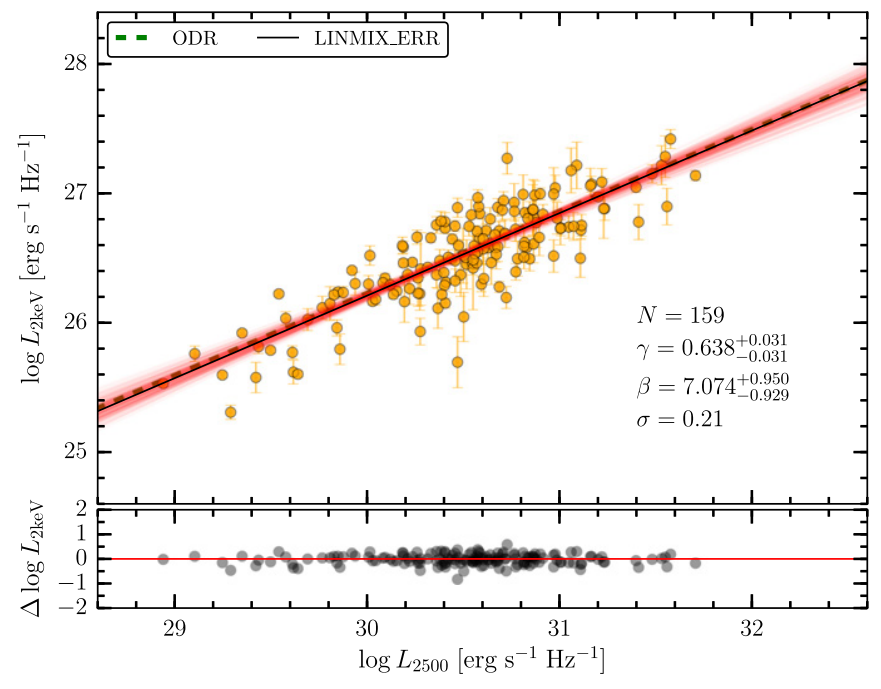

Figure 6. Keys as in Figure 3 for the selected quasar sample with multiple $\mathrm{X}$-ray detections. See Section 4 for details. The X-ray fluxes are estimate as the average between the 1st and 2nd $X M M$ longest exposure.

XMMSUSS2.1 catalog along with their uncertainties. The OM set of filters covers the optical and part of the UV wavelength range: $V, B, \quad U, \quad U V W 1, \quad U V M 2, \quad U V W 2$ with effective wavelengths 5430, 4500, 3440, 2910, 2310, and $2120 \AA$. Galactic reddening has been taken into account: we used the selective attenuation of the stellar continuum $k(\lambda)$ taken from Prevot et al. (1984) with $R_{\mathrm{V}}=2.7$. Galactic extinction is estimated from Schlegel et al. (1998) for each object. We derived total luminosities at the rest-frame frequency of the filter. The data for the SED computation from mid-infrared to UV (upper limits are not considered) were then blueshifted to the rest-frame.

Absorption from intergalactic $H i$, blueward of Ly $\alpha$ emission in the rest frame AGN SED, reduces the source flux both in the Lyman series (producing the so-called $\mathrm{Hi}$ forest), and in the Lyman continuum at rest $\lambda<912 \AA$ (e.g. Moller \& Jakobsen 1990). The $L_{2500}$ values extrapolated from rest-frame photometry bluer than Ly $\alpha$ could then be underestimated. In the SED construction we have been rather conservative and we have neglected all rest-frame data at wavelengths shorter than $1500 \AA(\log \nu \mathrm{Hz}>15.30)$. In the case of two data points inbetween $1500 \AA$ (but still at $\lambda<912 \AA$ ), we excluded the second entry at $\lambda<1500 \AA$.

In the case $2500 \AA$ were covered by no less than two data points, the $L_{2500}$ values are extracted from the rest-frame SEDs in the $\log \nu-\log \nu L(\nu)$ plane. If the SED is constructed by two data points (or more), but they do not cover $2500 \AA$, luminosities are extrapolated by considering the last (first) two photometric data points. Uncertainties on monochromatic luminosities from interpolation (extrapolation) are computed as in Equation (1). In the case the SED had only one data point in the wavelength range $1500-4000 \AA$, we computed $L_{2500}$ by employing a fixed optical slope $\gamma \sim 0.61 \pm 0.01$ (Telfer et al. 2002; Shull et al. 2012; Lusso et al. 2015; Stevans et al. 2014). The uncertainty on the slope has been properly propagated.

We estimated monochromatic rest-frame luminosities at $2500 \AA$ for 118 quasars in the selected X-ray detected sample, which are in very good agreement with the ones we considered in our analysis although with a moderate dispersion $(\sim 0.2 \mathrm{dex})$.
Such dispersion is mostly due to the different methodology used to compute the two optical-UV luminosities. Shen et al. luminosities are estimated through a continuum fit, while the OM ones still have some emission line contribution. Thus, the true optical-UV variability should be much lower, although it is not straightforward to quantify to what degree. We then computed slope, intercept, and dispersion (using LINMIX_ERR) of the $L_{2500}-L_{2} \mathrm{keV}$ relation for the 118 quasars within the clean X-ray detected sample with simultaneous $L_{2500}$ values finding $\gamma=0.697 \pm 0.040, \beta=5.199_{-1.211}^{+1.201}$, and a dispersion of 0.25 dex. The same procedure has been done by replacing the optical luminosities with the non-simultaneous values finding $\gamma=0.694 \pm 0.039, \beta=5.333 \pm 1.180$, and $\sigma=$ $0.25 \mathrm{dex}$, which is fully consistent with the simultaneous fit.

This finding is consistent with the work done by Vagnetti et al. (2010). They have analyzed simultaneous observations for 241 quasars (46 sources with multi-epoch data and 195 objects with single-epoch observations) having the X-ray/ optical-UV information from the first releases of both $X M M$ and OM catalogs (see their Section 2 for details). The observed dispersion in their simultaneous data was not significantly smaller than what previously found in non-simultaneous studies, which indicates that the "artificial variability" introduced by the non-simultaneity was not the main source of dispersion.

\section{DISCUSSION AND CONCLUSIONS}

The observed $L_{2500}-L_{2} \mathrm{keV}$ relationship in quasars indicates that there is a good "coupling" between the disk, emitting the primary radiation, and the hot-electron corona, emitting X-rays. Earlier studies have found that the scatter on this relation is $\sim 0.35-0.4$ dex, which is a combination of measurement uncertainties, variability, and intrinsic dispersion due to differences in the AGN physical properties. We analyzed in depth the various sources of the observed dispersion on the $L_{2500}-L_{2 \mathrm{keV}}$ relationship for a sample of 2153 optically selected quasars with X-ray data from the 3XMM-DR5 source catalog.

Our study shows that, once a homogeneous quasar sample is selected, the observed dispersion on the $L_{2500}-L_{2 \mathrm{keV}}$ relationship, for a sample of 159 quasars with multiple observations, is $\sim 0.23$ dex considering the longest $\mathrm{X}$-ray exposure. If we instead average X-ray luminosity values, we found that the scatter further reduces to $\sim 0.21 \mathrm{dex}$. This result can be parametrized as follows

$$
\left\{\begin{array}{l}
0.23=\sqrt{\sigma_{\mathrm{X}}^{2}+\sigma_{\mathrm{I}}^{2}} \\
0.21=\sqrt{\frac{\sigma_{\mathrm{X}}^{2}}{2}+\sigma_{\mathrm{I}}^{2}}
\end{array}\right.
$$

where $\sigma_{\mathrm{X}}$ is the contribution of the observed X-ray variability to the dispersion, and $\sigma_{\mathrm{I}}$ denotes all other possible sources of scatter (e.g., uncertainties on X-ray calibrations, optical variability, intrinsic variability related to AGN physics). In other words, $\sigma_{\mathrm{I}}$ represent our ignorance on the observed dispersion. From the simple system above we have: $\sigma_{\mathrm{X}}=0.13$ (consistent with the findings discussed in Section 4) and $\sigma_{\mathrm{I}}=0.19$. Variability in the optical is on the order of $0.05 \mathrm{dex}$ (Kozłowski et al. 2010 and references therein), which gives us a residual scatter of $0.18 \mathrm{dex}$. This low scatter provides a 

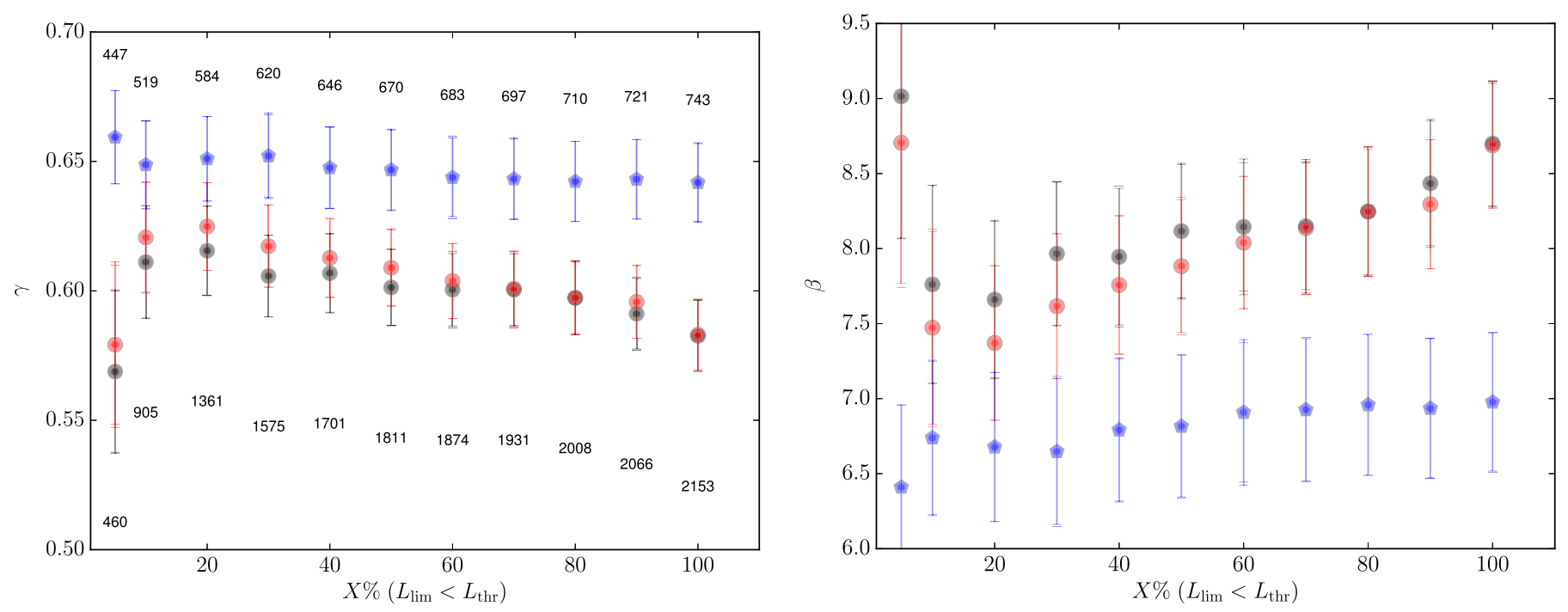

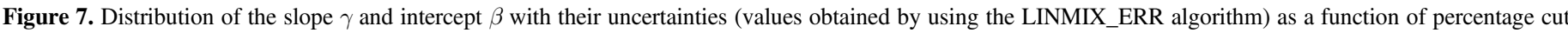

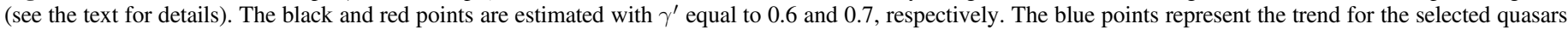
only with $\gamma^{\prime}$ equal to 0.6 . The number of objects that fulfill the selection in each slice is reported on top of the plotted values.

stringent observational constraint that any future self-consistent disk-corona models must explain. The tight $L_{2500}-L_{2 \mathrm{keV}}$ relationship discussed here also allows to accurately estimate the quasar X-ray luminosity for a given optical-UV luminosity, in order to define a standard range of soft X-ray emission for typical quasars (i.e., non-BAL/RL with minimum contamination of absorption), or vice-versa to easily identify peculiar objects (e.g., X-ray weak, strong RL).

Finally, establishing a tight correlation between $L_{2500}$ and $L_{2 \mathrm{kev}}$ for a statistically significant quasar sample over a wide range of redshifts and luminosities is the first step to robustly estimate cosmological parameters (Risaliti \& Lusso 2015). One of the main limitation of the standard candle approach lies in the large observed dispersion of the relation $(\sim 0.3 \mathrm{dex})$. Although the quasar sample analyzed here presents a remarkably tight $L_{2500}-L_{2 \mathrm{keV}}$ relationship, the low number of quasars, especially at high redshift (e.g., only 14 and 1 quasar have $z>3$ within the X-ray detected sample composed by 743 and 159 sources, respectively), allows us to obtain only loose constraints on $\Omega_{\mathrm{M}}$, and $\Omega_{\Lambda}$. XMM-Newton and Chandra observations of larger samples of high- $z$ quasars would better sample the Hubble diagram and provide tighter constraints on cosmological parameters.

In summary, our main findings are the following.

1. If we consider an optically selected quasar sample having minimum host-galaxy/reddening contamination and X-ray absorption, and reasonable $\mathrm{X}$-ray $\mathrm{S} / \mathrm{N}$ ratio (i.e., $>5$ ), the dispersion is much lower than what previously reported in the literature $(0.24$ dex against $0.35-0.4 \mathrm{dex})$. The slope and intercept are, overall, consistent (within their uncertainties) with $\sim 0.60-0.65$ and $\sim 7-8$, respectively, and they are almost independent on the quality cuts we applied.

2. Quasars having at least two X-ray observations in the cleaned data set (159 sources) typically vary with a standard deviation of fractional variation of about $30 \%$.

3. Based on our analysis of the correlation in a sub-sample with multiple observations, we measure a dispersion as low as 0.21 , and we estimate that the "intrinsic" dispersion, i.e., the dispersion not due to measurement statistical and/or systematic uncertainty, is lower than 0.19 .

4. The dispersion on the $L_{2500}-L_{2 \mathrm{keV}}$ relation estimated taking simultaneous data is not significantly smaller than what we find in the non-simultaneous sample. This indicates that the "artificial variability" introduced by the non-simultaneity is not the main source of dispersion.

We thank the anonymous referee for his/her useful comments and suggestions which have improved the clarity of the paper. We also thank Cristian Vignali for carefully reading our paper, Giorgio Lanzuisi for suggestions on quantifying X-ray variability, Fausto Vagnetti for clarifications about sample matching, and Andrea Comastri and Piero Ranalli for comments on the quasar sample with X-ray upper limits. For all catalog correlations we have used the Virtual Observatory software TOPCAT (Taylor 2005) available online (http://www.star.bris.ac.uk/ mbt/topcat/). This research has made use of data obtained from the 3XMM XMM-Newton serendipitous source catalog compiled by the 10 institutes of the XMM-Newton Survey Science Centre selected by ESA. This research has made use of the XMM-OM Serendipitous Ultra-violet Source Survey, which has been created at the University College London's (UCL's) Mullard Space Science Laboratory (MSSL) on behalf of ESA and is a partner resource to the 3XMM serendipitous X-ray source catalog. This research made use of matplotlib, a Python library for publication quality graphics (Hunter 2007). This work has been supported by the grant PRIN-INAF 2012. E.L. is grateful to Keele University for the hospitality.

\section{APPENDIX THE FLUX-LIMIT BIAS}

As already pointed out in Section 3, flux limited samples may be biased, and thus one needs to find an alternative method to obtain an (almost) unbiased sample even without the inclusion of censored data. To do so, we have sliced the main quasar sample of X-ray detected quasars (2155 objects) including only those sources that have a minimum X-ray 
luminosity ( $L_{\min }$, as estimated from the $F_{\min }$ at $2 \mathrm{keV}$, see Section 2.1) below a threshold defined as follows

$$
\log L_{\mathrm{thr}}=\gamma^{\prime} \log L_{2500}+\beta^{\prime},
$$

where $\gamma^{\prime}$ is assumed to be 0.6 and $\beta^{\prime}$ is a variable normalization. The normalization $\beta^{\prime}$ is defined so that the source fraction enclosed $\left(L_{2 \mathrm{keV}}<L_{\mathrm{thr}}\right)$ is in the range from $5 \%$ to $100 \%$. We then included the objects in the sample only if $L_{\text {min }}$ is below the detection limit given by Equation (5), thus regardless of the observed value of $L_{2} \mathrm{kev}$. We checked the effects of this cut varying the rejection fraction. The slope $\gamma$ and intercept $\beta$ (along with their uncertainties) of the $L_{2500}-L_{2 \mathrm{keV}}$ relation for each slice are estimated by using the LINMIX_ERR algorithm. We have also repeated the same approach with $\gamma^{\prime}=0.7$. Figure 7 shows the results of this experiment. Black and red points represent the findings for $\gamma^{\prime}=0.6$ and 0.7 , respectively. Blue points are the outcome considering $\gamma^{\prime}=0.6$ where we applied the quality cuts discusses in Section 3. If the flux-limit bias were strongly affecting the slope measurements in the X-ray detected quasar sample, we would have expected a flatter slope $(\gamma=0.7-0.8)$ for higher cuts $(X \% \lesssim 20)$. In other words, a decreasing of $\gamma$ as a function of $X \%$. On the other hand, the intercept $\beta$ must increase as a function of $X \%$. The regression parameters are, instead, not strongly dependent (within their uncertainties) either on the choice of $\gamma^{\prime}$ in Equation (5) or on the threshold. The $\gamma$ and $\beta$ values resulting from the slicing of the clean quasar sample are even more flatter than for the whole sample. Meaning that, even if the flux-limit bias may still be present, is not altering our findings significantly (both $\gamma$ and $\beta$ are statistically different at less than $2 \sigma$ ). This experiment ensure that our investigation of the luminosity relation can be performed even if only detections are considered.

\section{REFERENCES}

Avni, Y., \& Tananbaum, H. 1982, ApJL, 262, L17

Bechtold, J., Siemiginowska, A., Shields, J., et al. 2003, ApJ, 588, 119

Buckley, J., \& James, I. 1979, Biometrika, 66, 429 (http://biomet. oxfordjournals.org/content/66/3/429.abstract)

Chanan, G. A. 1983, ApJ, 275, 45

Dadina, M. 2008, A\&A, 485, 417

Feigelson, E. D., \& Nelson, P. I. 1985, ApJ, 293, 192

Gibson, R. R., \& Brandt, W. N. 2012, ApJ, 746, 54

Gibson, R. R., Jiang, L., Brandt, W. N., et al. 2009, ApJ, 692, 758

Haardt, F., \& Maraschi, L. 1991, ApJL, 380, L51
Haardt, F., \& Maraschi, L. 1993, ApJ, 413, 507

Hewett, P. C., \& Wild, V. 2010, MNRAS, 405, 2302

Hunter, J. D. 2007, CSE, 9, 90

Isobe, T., Feigelson, E. D., Akritas, M. G., \& Babu, G. J. 1990, ApJ, 364, 104

Isobe, T., Feigelson, E. D., \& Nelson, P. I. 1986, ApJ, 306, 490

Just, D. W., Brandt, W. N., Shemmer, O., et al. 2007, ApJ, 665, 1004

Kellermann, K. I., Sramek, R., Schmidt, M., Shaffer, D. B., \& Green, R. 1989, AJ, 98, 1195

Kelly, B. C. 2007, ApJ, 665, 1489

Komatsu, E., Dunkley, J., Nolta, M. R., et al. 2009, ApJS, 180, 330

Kozłowski, S., Kochanek, C. S., Udalski, A., et al. 2010, ApJ, 708, 927

Kriss, G. A., \& Canizares, C. R. 1985, ApJ, 297, 177

La Franca, F., Franceschini, A., Cristiani, S., \& Vio, R. 1995, A\&A, 299, 19

Lanzuisi, G., Ponti, G., Salvato, M., et al. 2014, ApJ, 781, 105

Lavalley, M., Isobe, T., \& Feigelson, E. 1992, in ASP Conf. Ser. 25, Astronomical Data Analysis Software and Systems I, ed. D. M. Worrall, C. Biemesderfer, \& J. Barnes (San Francisco, CA: ASP), 245

Lusso, E., Comastri, A., Vignali, C., et al. 2010, A\&A, 512, A34

Lusso, E., Worseck, G., Hennawi, J. F., et al. 2015, MNRAS, 449, 4204

Maccacaro, T., Gioia, I. M., Wolter, A., Zamorani, G., \& Stocke, J. T. 1988, ApJ, 326, 680

Moller, P., \& Jakobsen, P. 1990, A\&A, 228, 299

Page, M. J., Brindle, C., Talavera, A., et al. 2012, MNRAS, 426, 903

Prevot, M. L., Lequeux, J., Prevot, L., Maurice, E., \& Rocca-Volmerange, B. 1984, A\&A, 132, 389

Prochaska, J. X., Madau, P., O’Meara, J. M., \& Fumagalli, M. 2014, MNRAS, 438, 476

Richards, G. T., Strauss, M. A., Fan, X., et al. 2006, AJ, 131, 2766

Risaliti, G., \& Lusso, E. 2015, ApJ, 815, 33

Rosen, S. R., Webb, N. A., Watson, M. G., et al. 2015, A\&A, in press (arXiv:1504.07051)

Schlegel, D. J., Finkbeiner, D. P., \& Davis, M. 1998, ApJ, 500, 525

Shen, Y., Richards, G. T., Strauss, M. A., et al. 2011, ApJS, 194, 45

Shull, J. M., Stevans, M., \& Danforth, C. W. 2012, ApJ, 752, 162

Steffen, A. T., Strateva, I., Brandt, W. N., et al. 2006, AJ, 131, 2826

Stevans, M. L., Shull, J. M., Danforth, C. W., \& Tilton, E. M. 2014, ApJ, 794, 75

Strateva, I. V., Brandt, W. N., Schneider, D. P., Vanden Berk, D. G., \& Vignali, C. 2005, AJ, 130, 387

Tananbaum, H., Avni, Y., Branduardi, G., et al. 1979, ApJL, 234, L9

Tang, S. M., Zhang, S. N., \& Hopkins, P. F. 2007, MNRAS, 377, 1113

Taylor, M. B. 2005, in ASP Conf. Ser. 347, Astronomical Data Analysis Software and Systems XIV, ed. P. Shopbell, M. Britton, \& R. Ebert (San Francisco, CA: ASP), 29

Telfer, R. C., Zheng, W., Kriss, G. A., \& Davidsen, A. F. 2002, ApJ, 565, 773

Vagnetti, F., Turriziani, S., Trevese, D., \& Antonucci, M. 2010, A\&A, 519, A17

Vasudevan, R. V., \& Fabian, A. C. 2009, MNRAS, 392, 1124

Vignali, C., Brandt, W. N., \& Schneider, D. P. 2003, AJ, 125, 433

Watson, M. G., Auguères, J.-L., Ballet, J., et al. 2001, A\&A, 365, L51

Watson, M. G., Schröder, A. C., Fyfe, D., et al. 2009, A\&A, 493, 339

Wilkes, B. J., Tananbaum, H., Worrall, D. M., et al. 1994, ApJS, 92, 53

Young, M., Elvis, M., \& Risaliti, G. 2009, ApJS, 183, 17

Young, M., Elvis, M., \& Risaliti, G. 2010, ApJ, 708, 1388

Yuan, W., Siebert, J., \& Brinkmann, W. 1998, A\&A, 334, 498 